\title{
Editorial: Entomopathogens for Sustainable Food Production
}

\author{
Surendra K. Dara ${ }^{1}$, Robert W. Behle ${ }^{2 *}$ and Steven P. Arthurs ${ }^{3}$ \\ ${ }^{1}$ University of California Cooperative Extension, San Luis Obispo, CA, United States, ${ }^{2}$ Crop Bioprotection Research Unit, \\ United States Department of Agriculture, Agriculture Research Service, Peoria, IL, United States, ${ }^{3}$ BioBee USA, Tucker, GA, \\ United States
}

Keywords: entomopathogen, sustainable agriculture, food production, biological control, biopesticide

\section{Editorial on the Research Topic}

\section{Entomopathogens for Sustainable Food Production}

Sustainable agriculture is necessary for the long-term production of food and human welfare. Modern agricultural practices to maximize short-term profits can cause environmental stress. This stress in various forms may deplete natural resources while selecting for biological organisms that thrive in the altered agricultural environment. Examples of stress include degraded soil health measured as reduced organic matter and selection for pests that are resistant to pesticides. Clearly, improved agricultural practices are needed to maintain high levels of food production while reducing or eliminating biological stress from short-sighted production and pest control strategies.

Entomopathogens are microbial organisms that cause diseases in arthropod pests such insects, mites, and ticks. Categories of entomopathogens include bacteria, fungi, microsporidia, nematodes, and viruses. Under certain favorable circumstances, disease epizootics caused by entomopathogens naturally reduce some pest populations, especially when there is an overabundance or high density of susceptible hosts. Under agricultural conditions, unassisted epizootics can develop as pest densities increase, but often too late to prevent economic crop damage. Converting a natural entomopathogen into a biological control requires some form of manipulation, like importation to a new environment, conservation of condition favoring survival of the entomopathogen, or augmenting titers of the beneficial organism as with application of an entomopathogen biopesticide. Thus, improved scientific knowledge and technologies are needed to transition natural entomopathogens into microbial biopesticides.

Biopesticides based on entomopathogen support the goals of sustainable agriculture. The U.S. Code Title 7, Section 3103 defines sustainable agriculture as an integrated system of plant and animal production practices having a site-specific application that will over the long-term: (1) satisfy human food and fiber needs; (2) enhance environmental quality and the natural resource base upon which the agriculture economy depends; (3) make the most efficient use of non-renewable resources and on-farm resources and integrate, where appropriate, natural biological cycles and controls; (4) sustain the economic viability of farm operations; and (5) enhance the quality of life for farmers and society as a whole.

Entomopathogens contribute to integrated plant and animal food production systems by limiting damage caused by arthropods including insects, mites, and ticks. Research on entomopathogens has resulted in commercially successful pest control products and technologies that have been integrated in current food production systems. Several early biopesticides were developed many decades ago with the bacterium Bacillus thuringiensis (Bt) and remain on the market today to controlling lepidopteran, dipteran, and coleopteran pests. Technology expanded Bt's pest control through crop transformation that allowed plants to produce the bacterium's toxin in the plant cell, effectively reducing the number of chemical pesticide applications for many crops. 
In contrast to most broad-spectrum chemical insecticides, entomopathogens do not degrade environmental quality or adversely impact natural resources. Several factors favor environmental benefits of entomopathogens in agricultural pest control. For example, many entomopathogens are pathogenic to certain species or groups of arthropod pests. Due to the co-evolution with natural enemies and other beneficial arthropods, many are compatible with other biocontrol agents and beneficial arthropods. Thus, entomopathogens effectively reduce crop damage by pests without interfering with services provided by pollinators, predators and parasites.

When used as a part of integrated production systems, entomopathogens contribute to sustained efficacy of synthetic pesticides by interrupting selection for pests with resistance. Indirect benefits such as this support economic farm operations by maintaining multiple control practices to provide effective pest control.

Transitioning pest control from synthetic pesticides to entomopathogen biopesticides improves the quality of life for agriculture and the general public by reducing or eliminating the hazards and concerns often associated with applications of synthetic pesticides to crops. Using entomopathogens for pest control reduces concerns about pesticide residues as indicated by relatively benign restrictions on many biopesticide product labels for bacterial, fungal and baculovirus biopesticides including a 0-day-preharvest interval and a short 4-h restricted entry interval for application. Further, nematode-based biopesticides are exempt from US EPA registration requirements. These benign restrictions attest to the safety aspects associated with entomopathogens.

To summarize their benefits, entomopathogens support sustainable food production by addressing many concerns associated with the application of synthetic pesticides. Even though entomopathogens have many beneficial qualities for sustainable agriculture, they are not without challenges that must be addressed to provide for commercial success and grower adoption. For example, the production of the microbial agent is often more expensive when compared with synthetic pesticide agents. Some entomopathogens like fungi, bacteria, and nematodes are amenable to mass liquid-culture production techniques that offer relatively lower costs with increased scale. Others, such as baculoviruses, still require in vivo production techniques involving susceptible insects. These techniques are labor-intensive and difficult to scale up. Beyond production restrictions, the living entomopathogens in biopesticides often require additional considerations such as refrigerated storage to maintain viability between production and application, again adding cost.

The information provided in this Special Research Topic shares knowledge of the benefits from using entomopathogens for control of arthropod pests and attaining sustainable pest control. Progress in the development of entomopathogens as microbial control agents moves agriculture closer to sustainable food production.

Mention of trade names or commercial products in this publication is solely for the purpose of providing specific information and does not imply recommendation or endorsement by the U.S. Department of Agriculture. USDA is an equal opportunity provider and employer.

\section{AUTHOR CONTRIBUTIONS}

All authors contributed to the content and edits of this article.

Conflict of Interest: SA is employed by company BioBee USA.

The remaining authors declare that the research was conducted in the absence of any commercial or financial relationships that could be construed as a potential conflict of interest.

Copyright (C) 2021 Dara, Behle and Arthurs. This is an open-access article distributed under the terms of the Creative Commons Attribution License (CC BY). The use, distribution or reproduction in other forums is permitted, provided the original author(s) and the copyright owner(s) are credited and that the original publication in this journal is cited, in accordance with accepted academic practice. No use, distribution or reproduction is permitted which does not comply with these terms. 